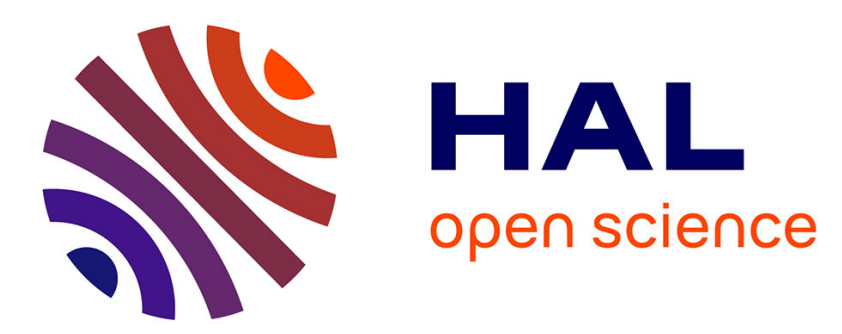

\title{
Judging beforehand the possibility of passing under obstacles without motion: the influence of egocentric and geocentric frames of reference
}

\author{
L. Bringoux, G. Robic, G. M. Gauthier, J. L. Vercher
}

\section{To cite this version:}

L. Bringoux, G. Robic, G. M. Gauthier, J. L. Vercher. Judging beforehand the possibility of passing under obstacles without motion: the influence of egocentric and geocentric frames of reference. Experimental Brain Research, 2008, 185 (4), pp.673-680. 10.1007/s00221-007-1194-7 . hal-01436033

\section{HAL Id: hal-01436033 https://hal.science/hal-01436033}

Submitted on 3 May 2018

HAL is a multi-disciplinary open access archive for the deposit and dissemination of scientific research documents, whether they are published or not. The documents may come from teaching and research institutions in France or abroad, or from public or private research centers.
L'archive ouverte pluridisciplinaire $\mathbf{H A L}$, est destinée au dépôt et à la diffusion de documents scientifiques de niveau recherche, publiés ou non, émanant des établissements d'enseignement et de recherche français ou étrangers, des laboratoires publics ou privés. 


\title{
Judging beforehand the possibility of passing under obstacles without motion: the influence of egocentric and geocentric frames of reference
}

\author{
L. Bringoux · G. Robic $\cdot$ G. M. Gauthier · \\ J. L. Vercher
}

Received: 12 June 2007/ Accepted: 20 October 2007/Published online: 8 November 2007

(C) Springer-Verlag 2007

\begin{abstract}
Previous studies have shown that the perception of the earth-based visual horizon, also named Gravity Referenced Eye Level (GREL), is modified by body tilt around a trans-ocular axis. Here, we investigated whether estimates of the elevation of a luminous horizontal line presented on a screen in otherwise darkness and estimates of the possibility of passing under are identically related to body tilt in absence of motion. Results showed that subjects overestimated the elevation of the projected line, whatever their body orientation. In the same way, subjects also overestimated their capacity of passing under the line. Both estimates appeared as a linear function of body tilt, that is, forward body tilt yielded increased overestimations, and backward body tilt yielded decreased overestimations. More strikingly, the linear effect of body tilt upon these estimates is comparable to that previously observed for direct GREL judgements. Overall, these data strongly suggest that the perception of the elevation of a visible obstacle and the perception of the ability of passing under in otherwise darkness shared common processes which are intimately linked to the GREL perception. The effect of body tilt upon these perceptions may illustrate an egocentric influence upon the semi-geocentric frame of reference required to perform the task. Possible interactions between egocentric and geocentric frames of reference are discussed.
\end{abstract}

L. Bringoux $(\bowtie)$ · G. Robic · G. M. Gauthier · J. L. Vercher UMR CNRS 6152 "Mouvement \& Perception",

Faculté des Sciences du Sport,

Université de la Méditerranée, 163,

avenue de Luminy CP 910, 13288 Marseille Cedex 9, France

e-mail: lionel.bringoux@univmed.fr
Keywords Spatial perception - Egocentric - Geocentric . Frame of reference - Gravity Referenced Eye Level . Body orientation

\section{Introduction}

Imagine you are stuck in your van, on a foggy day, waiting to enter a car park whose entrance is height restricted. You will probably ask yourself: "am I able to pass under the gate?" Perceiving the location of static obstacles in an impoverished visual environment is a complex task which is then crucial for avoiding collisions. The localization of an object in space may be achieved through different systems of coordinates named frames of reference (Howard 1982). Although visible surroundings may constitute a frame of reference for allocentric judgements, the body may define axes and planes relative to which egocentric judgements can be performed (Paillard 1991). For instance, the height of a gate may be either referred to some objects present in the visual field or to "eye level" (Matin and Li 1992). Although eye level is usually defined as a central norm for up and down egocentric localization in darkness (Matin and Li 1995), it may evoke two distinct spatial references, often undistinguished in the literature. One is the plane normal to the frontal plane of the head (HeadReferenced Eye Level or HREL) and the other is the plane normal to the direction of gravity (Gravity Referenced Eye Level or GREL; Stoper and Cohen 1989). Both references coincide when the observer stands stationary and erect but become different as soon as the observer is tilted. Although HREL judgements can be assessed in a pure egocentric frame of reference, GREL estimates must integrate a geocentric component (i.e., the horizontal plane). Therefore, GREL, also known as the earth-based visual horizon, 
can be considered a semi-geocentric reference (Bringoux et al. 2004, 2007).

The main purpose of the present experiment is to investigate whether the judgement of the elevation of an object (e.g., a horizontal line) and estimate of passing under in darkness share common mechanisms for which GREL constitutes the fundamental spatial reference.

Until recently, it was broadly accepted that specific sensory cues, mainly issued from vestibular and somatosensory inputs, were able to inform accurately about some geocentric directions of space (Benson 1990; Pozzo et al. 1990). This assumption mainly stems from the confusion between efficient postural control for stance conservation mediated by vestibulo-spinal pathways and higher perceptual graviceptive functions. Several experiments demonstrated, however, that the conscious estimation of the gravitational direction fundamentally differs from the perception and control of body orientation (Bringoux et al. 2003; Bronstein 1999). Nevertheless, as otolith organs and other somatic graviceptors were thought to provide accurate information about vertical and horizontal directions, GREL was assumed to be rather correctly estimated under normal circumstances (Schöne 1964; Tribukait and Eiken 2005).

In darkness, however, the subjective GREL appears slightly lower than the objective physical reference (MacDougall 1903; Stoper and Cohen 1986). It has also been shown that GREL perception is influenced by pitch tilts of the visual field (Li et al. 2001; Matin and Fox 1989; Matin and Li 1992; 1995; Stoper and Cohen 1989) and by modifications of the gravitoinertial force field strength (Cohen 1973; DiZio et al. 1997; Lackner and Graybiel 1980; Schöne 1964; Tribukait and Eiken 2005; Welch et al. 1996). More recently, GREL judgements in normogravity have been found linearly deviated towards body tilts ranging from $30^{\circ}$ backward to $20^{\circ}$ forward (Bringoux et al. $2004,2007)$. The quantity of this deviation reached $20 \%$ of the body tilt magnitude. This phenomenon, named "egocentric attraction", has been interpreted as a perceptual shift, in line with the idiotropic vector hypothesis stated for vertical estimation (Mittelstaedt 1983). The latter postulates the existence of a central tendency to bias the subjective vertical towards the direction of the observer's body Z-axis. Similarly, the tendency to shift GREL estimates towards HREL may illustrate an egocentric influence upon the semi-geocentric frame of reference required to perform the task. Subjects with bilateral vestibular deficit exhibit a comparable effect (Bringoux et al. 2007), suggesting that vestibular inputs are not determinant in counteracting this egocentric attraction. Comparable shifts when tilted have been reported for judgements of hand orientation relative to earth-fixed horizontal (Chelette et al. 1995) or judgements of the forearm orientation relative to earth-fixed vertical (Darling and Hondzinski 1999). However, as shifts in GREL estimates correspond to a modification of the perceived visual space (Schöne 1964), this may yield important consequences in the manner to which observers visually localize objects in an otherwise dark environment.

Several studies have shown that the localization of objects with respect to head-centric fixed planes (e.g., the transverse plane or the mid-sagittal plane of the head) was influenced by eye position, in the direction opposite to the eccentric gaze (Bock 1993; Lewald and Ehrenstein 2000). Alternatively, Poljac et al. (2005) demonstrated that the perceived elevation of objects relative to the "plane of regard" (defined by the interocular axis and the fixation point) is accurate, irrespective of eye and head orientation. Moreover, the gaze orientation was also found correctly estimated (Poljac and van den Berg 2005). This strongly suggests that the plane of regard constitutes a useful reference for accurate egocentric perception of objects' elevation. Therefore, it may be stated that a correct representation of the objects' location relative to the plane of regard is necessary for the transformation into a geocentric reference frame (Poljac and van den Berg 2005).

Within the general reference frame research area, the present study aims firstly at investigating whether the perception of objects' elevation into a geocentric reference frame is referred to GREL in absence of visual allocentric cues. In such a case, one would expect that the perceived elevation of an obstacle is modified by whole-body orientation, because the related reference (i.e., GREL) is linearly influenced by pitch tilts.

Secondly, this work aims at determining whether the perceived possibility of passing under a visible obstacle is also related to GREL perception in otherwise darkness. Such projective judgements would encounter the same dependency on body orientation as elevation estimates if one considers that: (1) the distance separating the obstacle from the observer (i.e., depth cue) is correctly estimated, (2) the observer internally represents the virtual displacement as horizontal (i.e., perpendicular to gravity), and (3) that body scheme-and specifically the perceived distance separating the eyes from the top of the head-is unmodified during body tilt.

\section{Methods}

Subjects

Twelve subjects (six males and six females; mean age $28 \pm 4.6$ years) with normal or corrected to normal vision participated in the experiments. They had no previous history of vestibular or other neurological symptoms. All 
gave informed consent, in compliance with the ethical laws which govern and regulate human experimentation in France.

\section{Apparatus}

The subjects were seated and firmly secured on a padded tilting chair by means of a shoulder harness (Fig. 1). The subjects' head, restrained with a headrest, was positioned such that the naso-occipital axis was orthogonal to the direction of gravity when the chair was vertically oriented. The axis of rotation of the tilting chair was coincident with the trans-ocular axis. This allowed in keeping eye level at the same height independently of the tilt magnitude. The chair could be tilted in pitch through a range extending from $+20^{\circ}$ backward to $-20^{\circ}$ forward. The random patterns of tilt induced angular accelerations well above the semicircular canals' threshold for tilt perception (defined at $0.3^{\circ} / \mathrm{s}^{2}$ by Benson 1990 ).

A laser pointer mounted on a fixed structure, positioned above the tilting chair was used to project a thin horizontal beam on a mirror. The pitch orientation of the mirror was adjustable by means of a servo-controlled galvanometer. The reflected beam was projected on a flat vertical screen of $2 \mathrm{~m}$ in height $\times 2.5 \mathrm{~m}$ in width, placed in front of the subjects, $2.28 \mathrm{~m}$ away from their eyes. The height of the luminous horizontal line, $2 \mathrm{~m}$ in width and $0.001 \mathrm{~m}$ in thickness, could be adjusted with a precision of $0.001 \mathrm{~m}$. Subjects held in hands a push button box for judgement settings. Galvanometer control and response recordings were performed by the ADwin-Pro system (Keithley ${ }^{\circledR}$ ) piloted via the Docometre ${ }^{\odot}$ software.

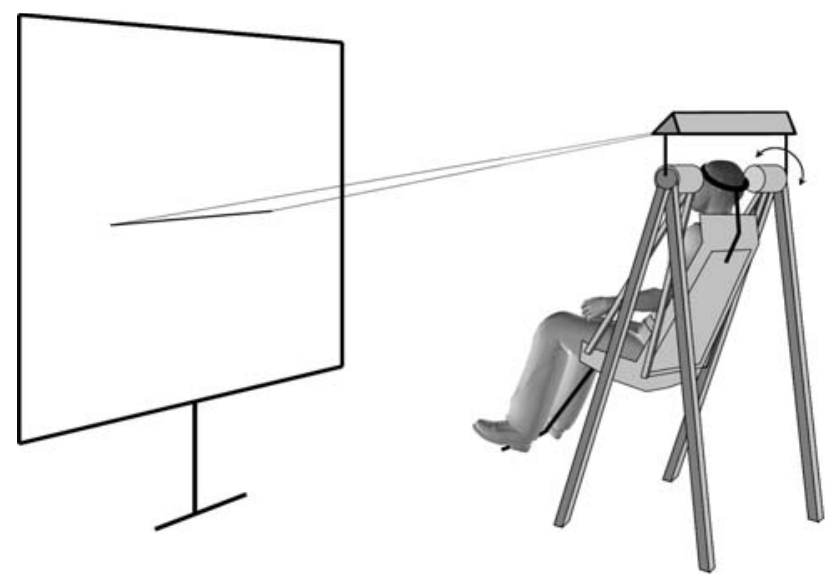

Fig. 1 Experimental set-up. The angular position of the platform could be set from $+20^{\circ}$ backward to $-20^{\circ}$ forward by rotation around the subjects' trans-ocular axis. The screen on which the thin luminous horizontal line was projected at different elevations was $2.28 \mathrm{~m}$ away from the observer's eyes
Procedure

The present experiment was divided into two counterbalanced sessions, lasting $45 \mathrm{~min}$ each. The first session required the subjects to judge the height of a luminous horizontal line relative to their earth-based visual horizon, presented as "where the sky meets the sea". Drawings and illustrations were also shown to avoid any confusion about the nature of the reference. In the second session, the subjects were asked to estimate whether they would be able to pass under the projected luminous line with their current body orientation. During this session, subjects were not told about any reference such as eye level or visual horizon for making their judgement. This clearly distinguishes the second perceptual task with respect to the former. For both sessions, five sagittal body orientations were randomly adjusted $\left(0^{\circ}\right.$; forward tilts: $-10^{\circ},-20^{\circ}$; backward tilts: $\left.+10^{\circ},+20^{\circ}\right)$. For each of these orientations, 10 luminous line elevations were randomly presented, ranging from $+20 \mathrm{~cm}$ upward to $-35 \mathrm{~cm}$ downward relative to eye level for session 1 and from $+35 \mathrm{~cm}$ upward to $-20 \mathrm{~cm}$ downward relative to eye level for session 2 .

A typical sequence of judgements unrolled as follows: The subjects were first rotated to the desired angle of orientation. This was followed by a $15 \mathrm{~s}$ period of rest, allowing the post-rotational effects issued from the semicircular canals stimulation to fade away (Benson 1990). Then, the luminous line was projected at a set height in the otherwise dark room, and the subjects were allowed to open their eyes and stare at it for $4 \mathrm{~s}$. Following this period of observation, subjects were asked to give a forced-choice response in a $3 \mathrm{~s}$ interval via the push buttons ("above the earth-based horizon" or "able to pass" with the right handheld button and "below the earth-based horizon" or "not able to pass" with the left hand-held button). Then, the subjects were requested to close their eyes for about $5 \mathrm{~s}$ while the luminous line was adjusted to a new height. Ten judgements (corresponding to 10 line elevations) were collected within a sequence executed at a given body orientation. Each sequence ended by a rotation of the tilting chair back to the vertical position, and the room was turned on for $5 \mathrm{~s}$ before a new sequence was launched. Four similar sequences, for a total of 200 judgements, were recorded in each session.

\section{Data processing}

Judgements were converted in binary values. A score of 1 was attributed to positive estimates, that is, when a line elevation was perceived higher than the earth-based horizon (session 1) or higher than the minimal height for passing under (session 2). Conversely, a score of 0 was 
attributed to negative estimates. A Probit model, using a non-linear regression analysis for dichotomic variables, enabled us to determine the probability $P$ that a line elevation be judged higher or lower than the considered reference. The Probit function (Eq. 1) was characterized by the following relation:

$p_{\mathrm{i}}=1 /\left(1+\left(\mathrm{C}\left({ }_{\mathrm{i}, \mathrm{j}}\right) / \mathrm{C}_{0}\right)^{\mathrm{n}}\right)$

where " $p_{\mathrm{i}}$ " is the probability of perceiving a line elevation higher than the related reference, "i" corresponds to the line number in the sequence, " $\mathrm{j}$ " to the trial number, " $\mathrm{C}_{0}$ " the line number for $P=0.5$ and "n" the slope of the tangent at the inflection point of the curve. The latter coefficient constitutes an estimation of the discrimination sensibility relative to the chosen increments. A repeated measures Analysis of Variance (ANOVA) was performed on " $n$ " values, to test any differences between sessions and body orientation conditions.

Line elevations obtained at $P=0.5$ via the psychometric function define thresholds for the perceived earth-based horizon, that is, the subjective GREL (session 1), and for the minimal height required for passing under obstacles (session 2). A repeated measures ANOVA was applied to these thresholds, calculated for each subject at each body orientation, to test any differences between sessions and body orientations. For convenience, thresholds were expressed as a vertical elevation (in $\mathrm{cm}$ ) relative to eye level. Finally, a linear regression analysis was conducted on the mean thresholds relative to the magnitude of body tilt, to establish the presence of a linear effect of body orientation upon estimates.

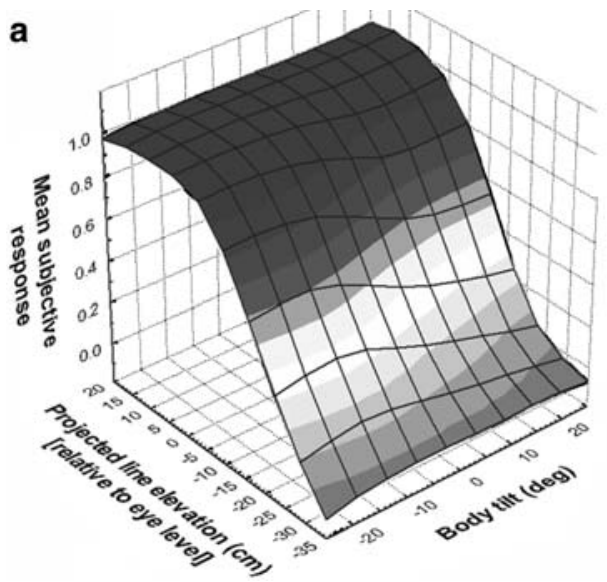

Fig. 2 Typical psychometric functions from a subject obtained via Probit non-linear regression analysis for the different body orientations in the two experimental sessions. The mean subjective responses corresponded to the mean perceptual scores obtained when the subject had to estimate that a particular line elevation was either higher (score $=1)$ or lower $($ score $=0)$ than Earth-based horizon (Session 1:

\section{Results}

Probit analysis

A non-linear regression analysis (Probit function) applied to raw judgements was used on each subject's data to specify the thresholds around which a line elevation was perceived higher or lower than the related reference (see Methods). Figure 2 shows the psychometric functions obtained for a subject at different body orientations.

To assess the discrimination sensibility of the Probit processing, a 2 session $\times 5$ body orientation $\left(-20^{\circ} ;-10^{\circ}\right.$; $\left.0^{\circ},+10^{\circ} ;+20^{\circ}\right)$ ANOVA was applied to the " $n$ " values (i.e., the slopes calculated at the inflection point of each function). Results showed that the discrimination sensibility did not differ, whatever the session $\left(F_{(1,11)}=0.45\right.$, $P=0.52)$ or the body orientation $\left(F_{(4,44)}=2.99\right.$, $P=0.11)$. The interaction between both factors was also non-significant $\left(\left(F_{(4,44)}=0.36, P=0.84\right)\right.$.

\section{Mean threshold comparisons}

The thresholds obtained via the Probit analysis were found notably lower than true eye level. In session 1 , the mean line elevation perceived at earth-based horizon (i.e., the subjective GREL) was $-10.5 \mathrm{~cm}$ relative to eye level, that is, subjects consistently overestimated the elevation of the projected line with respect to eye level. In session 2, the mean minimal height for passing under the line was $-1.12 \mathrm{~cm}$ relative to eye level. This means, for instance,

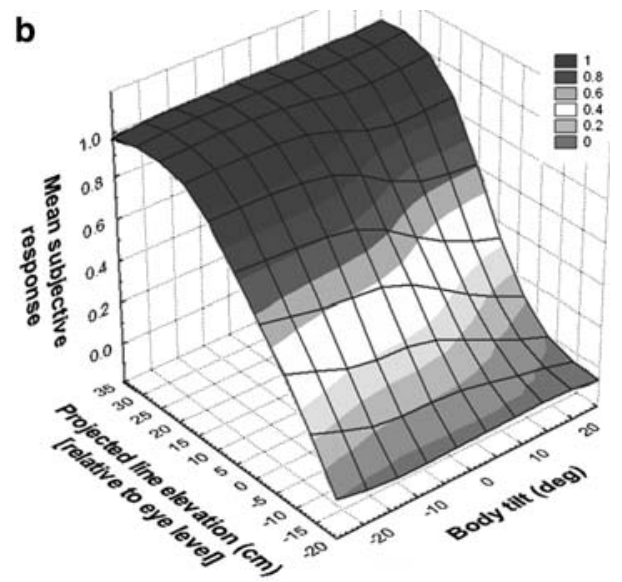

a) or than the minimal height for passing under (Session 2: b). The values of subjective responses extracted at $P=0.5$ from each Probit function correspond to the thresholds for Subjective GREL (session 1) and the perceived minimal height for passing under the line (session 2) 
that subjects judged they could pass under a line projected at eye level. In other words, they overestimated their capacity of passing under an obstacle, whatever their body orientation.

A 2 session $\times 5$ body orientation $\left(-20^{\circ} ;-10^{\circ} ; 0^{\circ}\right.$, $+10^{\circ} ;+20^{\circ}$ ) ANOVA conducted on the calculated thresholds revealed a significant difference between sessions $\left(F_{(1,11)}=20.56 ; P<0.01\right)$. The mean threshold corresponding to the perceived minimal height required for passing under obstacles was unsurprisingly higher than the subjective GREL. The mean difference between both thresholds $(9.5 \mathrm{~cm})$ was close to the physiological distance between eyes and upper head $(11 \mathrm{~cm})$.

The ANOVA also yielded a significant effect of body orientation $\left(F_{(4,44)}=8.06 ; P<0.01\right)$. A post hoc analysis (Newman-Keuls test) showed that the thresholds obtained at $+20^{\circ}$ of body tilt were significantly higher than those calculated at $0,-10^{\circ}$ and $-20^{\circ}(P<0.01)$. In the same way, thresholds obtained at $+10^{\circ}$ were significantly higher than those calculated at $-20^{\circ}(P<0.05)$. The interaction between both factors was non-significant $\left(F_{(4,44)}=0.21\right.$; $P=0.93)$, that is, the effect of body orientation was not different between sessions (Fig. 3).

Linear regression analysis on threshold estimates

The linear regression analysis applied to the thresholds obtained in both sessions with respect to body orientation (Fig. 3) showed a significant effect of body tilt upon the subjective GREL $\left(F_{(1,3)}=22.11 ; P<0.05\right)$ and upon the perceived minimal height for passing under obstacles $\left(F_{(1,3)}=65.85 ; P<0.01\right)$. The more the subjects were tilted forward (up to $-20^{\circ}$ ), the lower the thresholds, that is, the

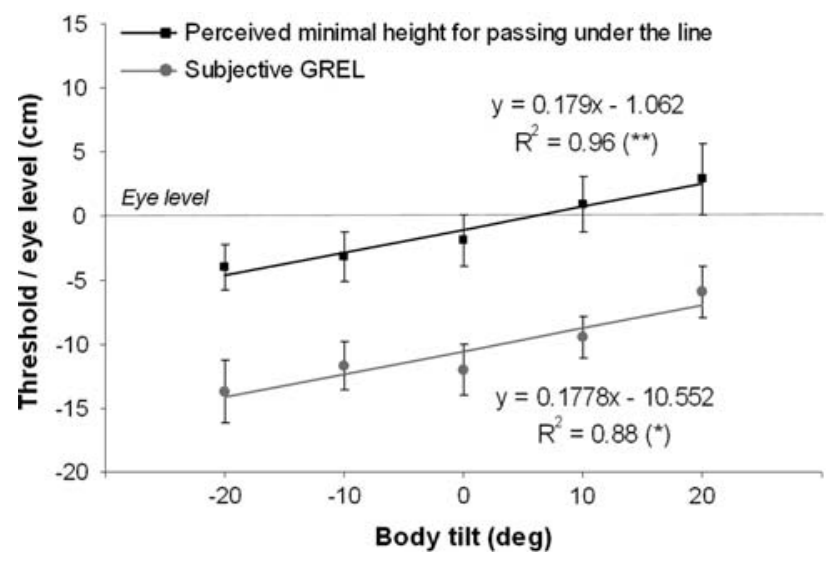

Fig. 3 Linear regression lines applied to the mean thresholds (plotted with $\pm \mathrm{SE}$ ) obtained for the different body orientations in the two experimental sessions. Subjective GREL and the perceived minimal height for passing under the line were found to be similarly and linearly related to body tilt more the subjects overestimated the elevation of an obstacle and their capacity of passing under. Conversely, the more the subjects were tilted backward (up to $+20^{\circ}$ ), the higher the thresholds, that is, the less the subjects overestimated the elevation of an obstacle and their capacity of passing under. Non-significant individual linear regressions observed in three subjects unauthorized the statistical comparison of slopes calculated for each regression line between sessions. Nevertheless, observation of the data and absence of significant interaction in the threshold comparison ANOVA strongly suggest that the linear effect of body orientation was similar in both sessions.

\section{Discussion}

The present study aimed at investigating whether the perceived elevation of a luminous obstacle in otherwise darkness and judgements of the capacity of passing under are identically influenced by body tilt. Both types of estimates were found inaccurate with respect to the true elevationsthat is, subjects overestimated the elevations of the projected line relative to GREL and their ability of passing under, whatever their body orientation. More strikingly, we showed that body tilt exerted a comparable linear influence on both estimates. The latter observation may indicate the existence of a common reference for judging the elevation of obstacles in a dark environment and the capacity of passing under in absence of any displacement.

\section{The GREL as a key reference}

The main finding of the present experiment is that body tilt yields a comparable influence on the perceived ability of passing under a luminous horizontal line as on elevation judgements relative to GREL. This strongly suggests that GREL constitutes a fundamental reference for estimating, without motion, the possibility of avoiding above-head obstacles when allocentric cues are not available. Changes observed on the subjective GREL during body tilt may then have a direct effect on the perceived ability of passing under obstacles, even when GREL is not explicitly specified as the reference to be used for the judgement (e.g., as in session 2). Former results discussed in the frame of the ecological theory of affordances (Gibson 1979; Warren 1984) already suggested that intrinsic information about object's elevation is scaled with reference to the perceivers' eyeheight (Mark 1987; Van Der Meer 1997). Specifically, when manipulating the optical texture convergence which led to illusory rising of the floor, a decrease in subjective eyeheight was observed, that in turn yielded a modification of "passability" judgements of apertures (Warren and Whang 1987). 
Similar eyeheight-scaled information was also found in the affordance of passing under a barrier for children and adults (Van Der Meer 1997).

However, such models of affordance were developed in presence of a structured visual field where information about environmental properties is naturally scaled in terms of some body dimensions of the observers (Mark 1987). Here, we demonstrate a similar effect of body tilt upon GREL judgements and estimations of the possibility of passing under obstacles without changing eyeheight-scaled information and in absence of structured visual field.

The fact that GREL perception is probably involved in the perceived ability of passing under obstacles implies three conditions to be fulfilled. The first one is that the distance separating the obstacle from the observer (i.e., depth cue) be correctly estimated. This is important, as an object located at a given angle above GREL will be perceived higher if its distance is overestimated. If fusional vergence (based on binocular disparity) is probably not a salient distance cue in the present study (involving a thin horizontal line projected on a screen), accommodation, in addition to the prior knowledge of the distance between the screen and the tilting chair, are good candidates to enable the perception of depth of the projected visual scene (Büttner and Büttner-Ennever 2005). The second required condition is that the observer internally represents the virtual displacement as horizontal. Once again, prior knowledge of the room configuration (e.g., horizontal floor) may facilitate the access to this information. A third condition implicitly underlies the direct link between the subjective GREL and the perceived ability of passing under obstacles. It requires that the perceived distance separating the eyes from the upper head be kept stable during body tilt. This assumption is supported by the constant gap between the two mean thresholds whatever body orientation, as illustrated by the parallel regression lines in Fig. 3. In addition, the mean difference between thresholds $(9.42 \pm 0.83 \mathrm{~cm})$ is close to the mean physiological distance between eyes and upper head, calculated at various body orientations $(11.3 \pm 0.86 \mathrm{~cm})$, suggesting an accurate and stable representation of body scheme for different body orientations or gravitational environments (Gurfinkel et al. 1993).

The idea that GREL may be regarded as a key reference in the perceived possibility of passing under obstacles is then mainly supported by the existence of a body tilt effect comparable to that observed for direct GREL estimates. The following part will discuss the potential origins of this body tilt effect.

Origins of the body tilt influence

Mittelstaedt (1983) postulates the existence of a central and idiosyncrasic tendency to shift the estimates towards the observer's own $Z$ axis, named "idiotropic vector". This is obviously the expression of an egocentric influence upon a geocentric judgement, close to the effect observed in the present study. The remaining question is which body part constitutes the predominant source of this egocentric attraction.

A first candidate could be the retinal meridian planes of the eyes (Poljac et al. 2005). Following this interpretation, such a geocentric estimate could be drawn towards the longitudinal retinal meridian (Wade and Curthoys 1997). In the pitch dimension, Poljac et al. (2005) showed that the plane of regard, containing the interocular axis and the line of sight, is a fundamental reference for egocentrically judging the elevation of objects, irrespective of head orientation. Further experiments need to be carried out to investigate whether the plane of regard is also predominantly involved in earth-based elevation judgements.

As a second candidate, the head may also play a major role in the reported egocentric attraction. Head stabilization relative to gravity while walking is thought to provide a stable egocentric reference for spatial perception (Pozzo et al. 1990). In the sagittal plane, several studies emphasized the involvement of the transverse plane of the head at eye level (HREL) as a reference for egocentric judgements (Stoper and Cohen 1989; Matin and Li 1992; 1995). Nevertheless, the specific role of this reference remains to be investigated for geocentric estimates collected in the pitch dimension.

At a higher level, the longitudinal whole-body axis has often been evoked as the main reference for egocentric attraction in earth-based judgements (Mittelstaedt 1983). Observations in microgravity strongly suggest that astronauts rely on the virtual line running from the head to the feet to determine the direction of up and down and orientations of objects in the spacecraft (Clément et al. 2007). Ito and Gresty (1996) demonstrated that a rostrocaudal trunk-and-leg axis is predominantly used as a reference for SVV settings in the sagittal plane when the body is tilted backward. Changing the posture of a seated human subject (e.g., extended or bended legs) would help determine the relative weight of this reference in the perceptual process yielding earth-based spatial orientation and localization.

Whatever the main body axes involved in the body tilt effect, our results support the hypothesis of an interaction between egocentric and geocentric frames of reference. In this context, tasks and/or environmental requirements could not only produce switches between frames of references (Ghafouri et al. 2002), but also mutual influences and partial overlapping. Several studies indeed suggest the existence of an intermediate state where the frame of reference normally required to adequately perform the task is distorted by a concurrent one (Heath et al. 2007; Neggers et al. 2005). Neural correlates of interactions between 
egocentric and allocentric frames of reference have been found in the right posterior parietal and right ventral premotor cortex (Fink et al. 2003; Committeri et al. 2004). Still, further experiments need to be conducted to isolate the neurophysiological locus of the interaction between egocentric and geocentric frames of reference emphasized in the present work.

\section{Conclusion}

Our study strongly supports the idea of common perceptual processes for judging, without motion, the elevation of a luminous object in otherwise darkness and the possibility of passing under. Both estimates may be based on the perception of the subjective GREL, a semi-geocentric reference whose perception may be biased towards some egocentric components, as attested by the linear influence of body tilt upon these judgements. Overall, our results may be of value for preventing misperceptions regarding the judged elevation of objects one is to pass under. For instance, the global-and potentially damaging-overestimation of the perceived possibility of passing under obstacles reported in the present experiment may be reduced when tilting observers backward. At least, one must be aware of the influence of postural orientation when one is required to pass under a gate at the entrance of a car park in deteriorated weather conditions.

Acknowledgments The authors are grateful to Franck Buloup for technical expertise and to Alexandra Chardenon and Thomas Shipley for fruitful discussions and constructive comments.

\section{References}

Benson AJ (1990) Sensory functions and limitations of the vestibular system. In: Warren R, Wertheim AH (eds) Perception and control of self-motion. Erlbaum, Hillsdale, pp 145-170

Bock O (1993) Localization of objects in the peripheral visual field. Behav Brain Res 56(1):77-84

Bringoux L, Nougier V, Barraud PA, Marin L, Raphel C (2003) Contribution of somesthetic information to the perception of body orientation in the pitch dimension. Q J Exp Psychol A 56(5):909-923

Bringoux L, Tamura K, Faldon M, Gresty MA, Bronstein AM (2004) Influence of whole-body pitch tilt and kinesthetic cues on the perceived gravity-referenced eye level. Exp Brain Res 155(3):385-392

Bringoux L, Mezey LE, Faldon M, Gresty MA, Bronstein AM (2007) Influence of pitch tilts on the perception of gravity-referenced eye level in labyrinthine defective subjects. Neuropsychologia 45(2):350-356

Bronstein AM (1999) The interaction of otolith and proprioceptive information in the perception of verticality. The effects of labyrinthine and CNS disease. Ann N Y Acad Sci 871:324-333
Büttner U, Büttner-Ennever JA (2005) Present concepts of oculomotor organization. Prog Brain Res 151:1-42

Chelette TL, Martin EJ, Albery WB (1995) The effect of head tilt on perception of self-orientation while in a greater than one $G$ environment. J Vestib Res 5(1):1-17

Clément G, Arnesen TN, Olsen MH, Sylvestre B (2007) Perception of longitudinal body axis in microgravity during parabolic flight. Neurosci Lett 413(2):150-153

Cohen M (1973) Elevator illusion: influences of otolith organ activity and neck proprioception. Percept Psychophys 14:318-322

Committeri G, Galati G, Paradis A, Pizzamiglio L, Berthoz A, LeBihan D (2004) Reference frames for spatial cognition: different brain areas are involved in viewer-, object-, and landmark-centered judgments about object location. J Cogn Neurosci 16(9):1517-1535

Darling WG, Hondzinski JM (1999) Kinesthetic perceptions of earthand body-fixed axes. Exp Brain Res 126(3):417-430

DiZio P, Li W, Lackner JR, Matin L (1997) Combined influences of gravitoinertial force level and visual field pitch on visually perceived eye level. J Vestib Res 7(5):381-392

Fink GR, Marshall JC, Weiss PH, Stephan T, Grefkes C, Shah NJ, Zilles K, Dieterich M (2003) Performing allocentric visuospatial judgments with induced distortion of the egocentric reference frame: an fMRI study with clinical implications. Neuroimage 20(3): $1505-1517$

Ghafouri M, Archambault PS, Adamovich SV, Feldman AG (2002) Pointing movements may be produced in different frames of reference depending on the task demand. Brain Res 929(1):117128

Gibson JJ (1979) The ecological approach of visual perception. Houghton Mifflin, Boston

Gurfinkel VS, Lestienne F, Levik YuS, Popov KE (1993) Egocentric references and human spatial orientation in microgravity. I. Perception of complex tactile stimuli. Exp Brain Res 95(2):339342

Heath M, Nelly K, Binsted G (2007) Allocentric visual cues influence online limb adjustments. Motor Control 11(1):54-70

Howard I (1982) Human visual orientation. Wiley, New-York

Ito Y, Gresty MA (1996) Shift of subjective reference and visual orientation during slow pitch tilt for the seated human subject. Brain Res Bull 40(5-6):417-421

Lackner JR, Graybiel A (1980) Visual and postural motion aftereffects following parabolic flight. Aviat Space Environ Med 51(3):230-233

Lewald J, Ehrenstein WH (2000) Visual and proprioceptive shifts in perceived egocentric direction induced by eye-position. Vision Res 40(5):539-547

Li W, Dallal N, Matin L (2001) Influences of visual pitch and visual yaw on visually perceived eye level (VPEL) and straight ahead (VPSA) for erect and rolled-to-horizontal observers. Vision Res 41(22):2873-2894

MacDougall R (1903) The subjective horizon. Psychol Rev 4:145-166

Mark LS (1987) Eyeheight-scaled information about affordances: a study of sitting and stair climbing. J Exp Psychol Hum Percept Perform 13(3):361-370

Matin L, Fox CR (1989) Visually perceived eye level and perceived elevation of objects: linearly additive influences from visual field pitch and from gravity. Vision Res 29(3):315-324

Matin L, Li W (1992) Visually perceived eye level: changes induced by a pitched-from-vertical 2-line visual field. J Exp Psychol Hum Percept Perform 18(1):257-289

Matin L, Li W (1995) Multimodal basis for egocentric spatial localization and orientation. J Vestib Res 5(6):499-518

Mittelstaedt H (1983) A new solution to the problem of the subjective vertical. Naturwissenschaften 70(6):272-281 
Neggers SFW, Schölvinck ML, van der Lubbe RHJ, Postma A (2005) Quantifying the interactions between allo- and egocentric representations of space. Acta Psychol (Amst) 118(1-2):25-45

Paillard J (1991) Motor and representational framing of space. In: Paillard J (eds) Brain and space. Oxford University Press, Oxford, pp 163-182

Poljac E, van den Berg AV (2005) Localization of the plane of regard in space. Exp Brain Res 163(4):457-467

Poljac E, Lankheet MJM, van den Berg AV (2005) Perceptual compensation for eye torsion. Vision Res 45(4):485-496

Pozzo T, Berthoz A, Lefort L (1990) Head stabilization during various locomotor tasks in humans. I. Normal subjects. Exp Brain Res 82(1):97-106

Schöne H (1964) On the role of gravity in human spatial orientation. Aerosp Med 35:764-772

Stoper AE, Cohen MM (1986) Judgments of eye level in light and in darkness. Percept Psychophys 40(5):311-316

Stoper AE, Cohen MM (1989) Effect of structured visual environments on apparent eye level. Percept Psychophys 46(5):469-475
Tribukait A, Eiken O (2005) Perception of the head transversal plane and the subjective horizontal during gondola centrifugation. Percept Psychophys 67(3):369-382

Van Der Meer ALH (1997) Visual guidance of passing under a barrier. Early Dev Parent 6:149-158

Wade SW, Curthoys IS (1997) The effect of ocular torsional position on perception of the roll-tilt of visual stimuli. Vision Res 37(8): 1071-1078

Warren WH (1984) Perceiving affordances: visual guidance of stair climbing. J Exp Psychol Hum Percept Perform 10:683-703

Warren WH, Whang S (1987) Visual guidance of walking through apertures: body-scaled information for affordances. J Exp Psychol Hum Percept Perform 13:371-383

Welch RB, Cohen MM, DeRoshia CW (1996) Reduction of the elevator illusion from continued hypergravity exposure and visual errorcorrective feedback. Percept Psychophys 58(1):22-30 